\title{
Effect of Ultrasound Guided Bilateral Greater Occipital Nerve Block on Serum Calcitonin Gene Related Peptide (CGRP) in Chronic Migraine
}

Mahmoud Haroun, Ali Soliman, Ramez Mostafa, Ahmed Elsadek, Randa Mohamed, Shahenaz Mohamed, Abdelrahman Atef* Department of Neurology - Ain Shams University.

*Corresponding Author: Abdelrahman Atef, Department of Neurology - Ain Shams University.

Received date: October 06, 2021; Accepted date: October 22, 2021; Published date: October 29, 2021

Citation: Antonis Theofilidis, Filipos Kargopoulos (2021) Effect of Ultrasound Guided Bilateral Greater Occipital Nerve Block on Serum Calcitonin Gene Related Peptide (CGRP) in Chronic Migraine. J. Neuroscience and Neurological Surgery. 10(1); DOI:10.31579/2578-8868/212

Copyright: (c) 2021 Abdelrahman Atef, This is an open-access article distributed under the terms of The Creative Commons Attribution License, which permits unrestricted use, distribution, and reproduction in any medium, provided the original author and source are credited

\begin{abstract}
:
Background: The trigeminal ganglion plays a key role in primary headache pathophysiology. Calcitonin gene-related peptide (CGRP) and CGRP receptors are expressed in trigeminal neurons that form C-fibers and A-fibers, respectively. In migraine attacks, there is release of CGRP into the cranial venous outflow, in refractory headache to conventional pharmacologic management, minimally invasive techniques such as greater occipital nerve block (GONB) are feasible for pain relief, and help to decrease the frequency of the attacks, Studies on the ultrasound (US) guided GON injection technique have emphasized that this technique has a higher success rate and should allow for a more precise block of the nerve. Our study will be concerned by correlation of CGRP level as a biomarker for effectiveness and responders of us guided GON block in chronic migraine (CM).
\end{abstract}

Methods: twenty patients diagnosed with chronic migraine were recruited in this study. All participants underwent ultrasound-guided bilat. GONB by $40 \mathrm{mg}$ triamcinolone and $1 \mathrm{cc}$ leidocaine using a portable ultrasound system with a $7-13 \mathrm{MHz}$ multifrequency transducer, blood samples were collected from antecubital vein immediately before and three to five weeks after injection clinical response was evaluated using headache diaries

Results: CGRP levels after ultrasound guided GONB (median, $40 \mathrm{pg} / \mathrm{mL}$; range, 25-60) were significantly lower as compared with CGRP levels obtained before GONB (median, 145 pg/mL; range, 60-380; P =0.001). Pretreatment CGRP levels in non-responders $(310 \mathrm{pg} / \mathrm{mL})$ were significantly higher than those seen in responders being in poor responders less than $50 \%$ improvement $(135 \mathrm{pg} / \mathrm{ml})$ and good responders $(140 \mathrm{pg} / \mathrm{mL} ; \mathrm{P}=0.003)$. One month after treatment. A number of demographic factors, clinical features, and comorbidities were not different in responders as compared with those of nonresponders.

Conclusion: These results suggests that interictal CGRP levels can be of help in predicting the response to GONB and suggest that the mechanism of action of GONB in CM is the reversal of sensitization as a result of the inhibition of CGRP release still more studies needed to highlight CGRP role with GONB

Key Words: chronic migraine; GONB; CGRP

\section{Introduction}

Migraine is a common, multifactorial, neurovascular disorder with major individual and societal effects. ( Goadsby et al.,2011)

Migraine affects roughly $15 \%$ of people and is typically characterised by disabling episodes of severe headache accompanied by nausea, vomiting, and hypersensitivity to light, sound, and smell for up to 3 days (migraine without aura).( Olesen et al.,2013).

In a third of patients, attacks might be associated with transient focal neurological aura symptoms (migraine with aura); it has been suggested that migraine with and without aura are distinct disorders. Once a migraine attack has started, the mechanisms underlying migraine aura and headache are reasonably well understood. Aura is most likely caused by cortical spreading depression, and headache by activation of the trigeminovascular system and associated release of CGRP. (Messlinger et al.,2011)

Migraine headache is caused by activation of the trigeminovascular system (TVS) The TVS consists of nociceptive trigeminal sensory afferents surrounding cranial blood vessels. Upon activation of these perivascular trigeminal afferents, the signal travels through the trigeminal ganglion to neurons in the trigeminocervical complex, using CGRP as the main neurotransmitter. The signals are then relayed to the thalamus; 
because all nociceptive inputs are integrated through this structure, it has been named the pain matrix of the brain.( Noseda et al.,2013)

Modulation of the signal occurs through extensive connections with brainstem regions such as the periaqueductal gray and the locus coeruleus.( Akerman et al.,2011)

GON block intervention is found to substantially reduce pain intensity and analgesic medication consumption for migraine patients, with no increase in adverse events. GON block intervention should be recommended to be administrated in migraine patients (zhang et al., 2018).

Once the headaches become refractory to conventional pharmacologic management, minimally invasive techniques such as peripheral nerve blocks are feasible for pain relief, and help to decrease the frequency of the attacks.Various studies have demonstrated that peripheral nerve blocks are safe and effective for the treatment of a variety of headache disorders. These techniques not only provide adequate analgesia but can also help to decrease systemic side effects of pharmacological therapy (Soto et al.,2012).moreover it was found that The US guided GON injection technique has a higher success rate and might allow a more precise block of the nerve (Vanderhoek et al., 2013).

The aim of this study was to to assess the effect of ultrasound guided bilateral GONB on CGRP levels in peripheral blood and outcome in chronic migraine (CM).

\section{Methods}

This study consecutively recruited 20 participants diagnosed with chronic migraine from headache outpatient clinics at Neurology Department of Ain Shams University Hospitals in the city of Cairo, from August 2018 through March 2021.

This was an interventional study with a convenient sample. Inclusion criteria of our sample were patients with the age above 18 years. Written informed consent was obtained from all participants. Exclusion criteria were patients with severe systemic diseases, infection or injury in occiput and patients with allergy to any of substances used in injection All patients were evaluated by $\bullet$ Clinical history (Detailed history including personal data, past history, history of current illness, antimigraine treatment used). All patients underwent ultrasound-guided bilateral GONB by $40 \mathrm{mg}$ triamcinolone and $1 \mathrm{cc}$ leidocaine using a portable ultrasound transducer.
The patient were asked to lie prone on the table. To locate the nerve we searched for the occipital artery in the medial one-third of the superior nuchal line between the occipital tubercle and mastoid process. The scalp was cleaned with iodine; GONB was performed by applying the injection to the medial of the artery. A needle was advanced beneath the lateral border of the probe using real-time ultrasound guidance and an in-plane technique ,Sampling :Blood samples were obtained after an overnight fast before initiating blockade and one month later to avoid the effect of circadian rhythms on biomarkers levels, blood samples were obtained between 9 and 11 a.m. Subjects didn't consume anti- inflammatory or analgesic medication in the previous 48 hours. A blood sample will be extracted from the nondominant forearm to measure serum CGRP, Efficacy was evaluated evaluated using: Diaries completed by patients in one month following the blockade,

\section{Statistical analysis}

The collected data was revised, coded and introduced to a PC using Statistical package for Social Science (SPSS 25, by IBM: Armonk; New York, US). Data were introduced and suitable analysis was done according to the type of data obtained for each parameter. Mean, standard deviation $( \pm \mathrm{SD})$ and range for parametric numerical data, while median and interquartile range (IQR) for non-parametric numerical data. Frequency and percentage for non-numerical data. Chi-Square test was used to assess the relationship between two qualitative variables. Student $\mathrm{T}$ Test was used to investigate the statistical significance of the difference between two study group means. ANOVA test was used to investigate the statistical significance of the difference between more than two study group means. Correlation analysis (using Spearman's rho and Pearson method) to assess the strength of association between two quantitative variables. $\mathrm{P}$-value level of significance was defined as $\mathrm{P}>0.05$ is nonsignificant, $\mathrm{P}<0.05$ is significant, and $\mathrm{P}<0.01$ is highly significant.

\section{Results}

\section{Clinical data:}

Forty patients fulfilling the CM criteria were included in this study. Mean age of CM patients $31.125 \pm 7.387$, only $4(20 \%)$ were males and the rest are females 16 (80\%) (. By history, the average time that patients had CM was $7.225 \pm 2.869$ years. The main phenotypes and associated symptoms of the patients that were enrolled in this study are shown in Table 1.

\begin{tabular}{||l|l|c|c||}
\hline Phenotypes and associated symptoms & N & $\%$ \\
\hline \hline \multirow{3}{*}{ Laterality } & Unilateral & 18 & 45 \\
\cline { 2 - 4 } & Bilateral & 22 & 55 \\
\hline \multirow{3}{*}{ Photophobia } & Frontal & 7 & 17.50 \\
\cline { 2 - 4 } & Temporal & 23 & 57.50 \\
\cline { 2 - 4 } & Occipital & 10 & 25.00 \\
\hline \multirow{3}{*}{ Phonophobia } & No & 7 & 17.50 \\
\cline { 2 - 4 } & Yes & 33 & 82.50 \\
\hline \multirow{2}{*}{ Lacrimation } & No & 5 & 12.50 \\
\cline { 2 - 4 } & Yes & 35 & 87.50 \\
\hline \multirow{2}{*}{ Vertigo } & No & 18 & 45 \\
\cline { 2 - 4 } & Yes & 22 & 55 \\
\hline \hline
\end{tabular}

Table1: Summary of clinical characteristics of our patients with chronic migraine. 


\section{CGRP determinations and correlation with clinical response:}

CGRP levels after GONB (median, $40 \mathrm{pg} / \mathrm{mL}$; range, 25-60) were significantly lower as compared with CGRP levels obtained before GONB (median, $145 \mathrm{pg} / \mathrm{mL}$; range, 60-380; $\mathrm{P}=0.001$ ) (Figure. 1).

$17(85 \%)$ responded and the remaining 3 patients (15\%) did not notice any significant response to GONB, about 8 patients (40\%) showed good response while 9 patients $(45 \%)$ showed poor response . Pretreatment CGRP levels in non-responders $(310 \mathrm{pg} / \mathrm{mL})$ were significantly very high than that seen in responders $(135-140 \mathrm{pg} / \mathrm{mL} ; \mathrm{P}=0.001)$. One month after treatment (table 2).while CGRP level after GONB wasn't significantly correlated to clinical response.

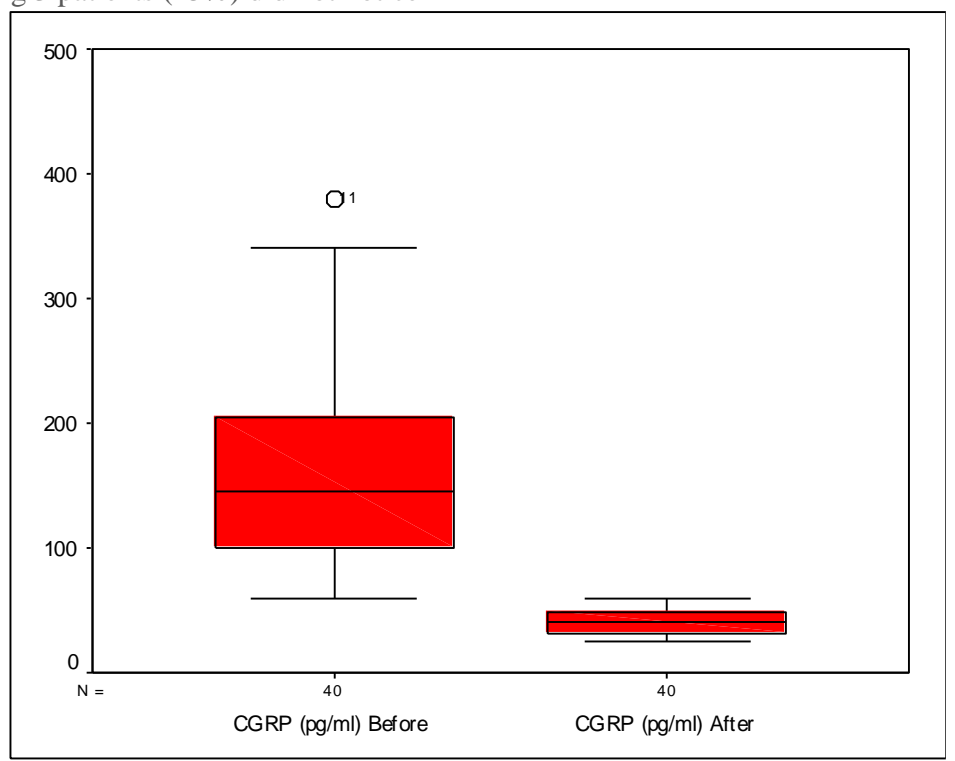

Figure 1: Box plot for the CGRP levels in chronic migraine patients before, and after GONB.

\begin{tabular}{|c|c|c|c|c|c|}
\hline \multirow{2}{*}{\multicolumn{2}{|c|}{ Diary }} & \multirow{2}{*}{ Median } & \multirow{2}{*}{ IQR } & \multicolumn{2}{|c|}{ Kruskal-Wallis Test } \\
\hline & & & & 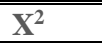 & P-value \\
\hline \multirow{3}{*}{$\begin{array}{l}\text { CGRP }(\mathrm{pg} / \mathrm{ml}) \\
\text { Before }\end{array}$} & No Response & 310 & $262.5-350$ & \multirow{3}{*}{11.839} & \multirow{3}{*}{$0.003^{*}$} \\
\hline & Poor Response & 135 & $100-200$ & & \\
\hline & Good Response & 140 & $80-150$ & & \\
\hline \multirow{3}{*}{$\begin{array}{l}\text { CGRP }(\mathrm{pg} / \mathrm{ml}) \\
\text { After }\end{array}$} & No Response & 44 & $38.75-50$ & \multirow{3}{*}{1.099} & \multirow{3}{*}{0.577} \\
\hline & Poor Response & 41 & $31-45.75$ & & \\
\hline & Good Response & 36 & $32.25-50$ & & \\
\hline
\end{tabular}

Table 2: CGRP levels before and after and its correlation to response to GONB

\section{Discussion:}

An increasedCGRP level has been proposed as the first biomarker to aid in obtaining a more objective diagnosis of $\mathrm{CM}$, in the context of a patient with daily or almost daily headaches and a history of migraine. CernudaMorollón, E. et al. (2013) We show here that treatment with GONB significantly decreases interictal CGRP levels measured in peripheral blood samples in patients with CM.These results contribute to clarifying the mechanism of action of GONB in CM and suggest that the measurement of interictal CGRP levels can be of help in predicting the response to GONB.

As regard headache days we considered decrease after injection by $>50 \%$ good response ,Less than $50 \%$ poor response and if no difference considered no response, our study showed that there was statistical significant reduction in headache days before and after injection with the mean $(19.35 \pm 3.1)$ before and after block it was (12.30 \pm 5.46$)$ with $40 \%$ showing good response, $45 \%$ poor response and $15 \%$ showing no response, Similar results were found in study by Ashkenazi and colleagues (Ashkenazi et al., 2008),

On the other hand Bovim and Sand (1992) examined the diagnostic value and effects of GON block and found only $6 \%$ in the migraine group showed good response and this may be attributed to blockade type as they used injection of 0.5 to $1.5 \mathrm{~mL}$ of lidocaine $2 \%$, with adrenaline 12.5 $\mu \mathrm{g} / \mathrm{mL}$, also using ultrasound guided technique added more accuracy and precise localization of the nerve which improved response among our patients.

We found that CGRP levels were highly elevated before GONB and this comes in concordance with study conducted by Goadsby PJ and Edvinsson L(1993) in which they conducted studied on both cats and humans with stimulation and found that CGRP level is elevated in migraneur patients and levels normalize after receiving sumatripatans and dihydroergotamine with relief of symptoms.

In our study we aimed to detect if there are changes in CGRP level after GONB and if it can be used as a biomarker predictor for response to GONB and we found that there was a significant reduction of CGRP level after GONB although There are no previous studies in the literarure measured the change in CGRP level after GONB but there were few studies measured CGRP level changes after botulinum toxin injection Cernuda-Morollón, etal.,(2015).

In our study we found that $45 \%$ of the patients showed good response ,40\% showed poor response and about $15 \%$ showed no response at all, 
here we show not only that CGRP levels are decreased after GONB, but also that this reduction correlates with its efficacy rate. In fact, CGRP levels decreased significantly, and very homogenously in almost each individuals but we found that pretreatment CGRP levels were high but in responders it was high but level didn't exceed 200

pg/ml (median, $135 \mathrm{pg} / \mathrm{mL}$; range, 100-200 in poor responder and median, $140 \mathrm{pg} / \mathrm{mL}$;range, 80-150 in good responders ; $\mathrm{P}=0.003$ ), while in nonresponders it was very high exceeding 200pg/ml (median, $310 \mathrm{pg} / \mathrm{mL}$; range, 262.5-350; $\mathrm{P}=0.003$ ) which again supports a role of CGRP levels in predicting response to this treatment, And these findings may support the need for repeated GONB to tackle resitant chronic migraine which didn't show initial response to GONB, Gul, H. L et al., (2017) conducted a study in which they included $44 \mathrm{CM}$ patients and randomly divide the patients into two groups, as group A (bupivacaine) and group B (placebo) , GON blockade was administered four times (once per week) and they found significant reduction in headache severity and frequency in group A compared to placebo group. Moreover In the RCT undertaken by Inan et al.,(2015) GON blocks were applied four times weekly. There was a significant decrease in the number of headaches and VAS scores.

In our study we found that percentage of change in both responders and non-responders was significant ( $\mathrm{P}=0.010$ ) while CGRP levels after injection was insignificant $(\mathrm{P}=0.577)$ raising the possibility that clinical improvement may be non CGRP mechanism these findings in nonresponders could be explained by limitations of this study, such as the absence of a control group, the fact that most of these patients remained on oral preventives, the relatively low numbers of nonresponders in our series, or the intrinsic and subjective variability of migraine, our results might indicate that some patients with a "CM" phenotype could suffer from other headaches (eg, psychogenic, tension-type headache or other secondary headaches) in which CGRP does not seem to be involved. This may be due to in some patients with migraine, pain could be secondary to a predominant activation of the parasympathetic arm of the TVS (Strassman AM et al ,1997). This could explain the fact that the efficacy of pure CGRP antagonists "gepants" seems to belower than that seen for triptans, which are known to inhibit both CGRP and VIP release by the TVS (Olesen J, etal 2009).

At the end we found that CGRP level before GONB may play a role as a predictor for response for the GONB as the higher the level of CGRP exceeding $200 \mathrm{pg} / \mathrm{ml}$ showed no response while lower levels than 200 $\mathrm{pg} / \mathrm{ml}$ showed respose ranging from poor $(<50 \%)$ and good response $(>50 \%)$ although both responders and non-responders showed significant reduction in CGRP levels and this may raise the importance of repeated GONB in non-responders while CGRP levels after injection didn't show significant correlation raising the possibility that improvement may be non CGRP mechanism moreover Our study has several limitations. First, the size of the sample is relatively small. Patients come from a selected clinical population. Many of them were using other preventive medications due to ethical reasons and this could work as a cofounder. The use of other drugs could also reduce levels of biomarkers related to inflammation, also a controlled trial would give more insight on CGRP changes.

Our current results suggest that the primary mechanism of action of GONB in CM is the reversal of peripheral and central sensitization as a result of the inhibition of the release of CGRP

The fact that we are the first study in the literature to assess the changes of CGRP levels with GONB gave us little chances to correlate our work with the others, hopefully in the future there will more studies regarding
CGRP changes with peripheral intervention as they are feasible with less side effect and affordable.

\section{List of Abbreviations}

CM............................Chronic migraine

GONB .............................Greater occipital nerve block

HIT6.............................Headache impact score

MIDAS...............................Migraine disability assessment scale

REM............................Rapid eye movement

SD .............................. Standard deviation

SPSS ............................ Statistical package for Social Science

TVS.

Trigemino vascul

VAS ............................... Visual analogue scale

\section{References}

1. Akerman, S., Holland, P. R., \& Goadsby, P. J. (2011). Diencephalic and brainstem mechanisms in migraine. Nature Reviews Neuroscience, 12(10), 570-584.

2. Bovim, G., \& Sand, T. (1992). Cervicogenic headache, migraine without aura and tension-type headache. Diagnostic blockade of greater occipital and supra-orbital nerves. Pain, 51(1), 43-48.

3. Cernuda-Morollón, E., Larrosa, D., Ramón, C., Vega, J., Martínez-Camblor, P., \& Pascual, J. (2013). Interictal increase of CGRP levels in peripheral blood as a biomarker for chronic migraine. Neurology, 81(14), 1191-1196.

4. Cernuda-Morollón, E., Ramón, C., Martínez-Camblor, P., Serrano-Pertierra, E., Larrosa, D., \& Pascual, J. (2015). OnabotulinumtoxinA decreases interictal CGRP plasma levels in patients with chronic migraine. Pain, 156(5), 820-824.

5. Diener, H. C., Dodick, D. W., Goadsby, P. J., Lipton, R. B., Olesen, J., \& Silberstein, S. D. (2012). Chronic migraineclassification, characteristics and treatment. Nature Reviews Neurology, 8(3), 162-171.

6. Goadsby, P. J., \& Edvinsson, L. (1993). The trigeminovascular system and migraine: studies characterizing cerebrovascular and neuropeptide changes seen in humans and cats. Annals of Neurology: Official Journal of the American Neurological Association and the Child Neurology Society, 33(1), 48-56.

7. Gul, H. L., Ozon, A. O., Karadas, O., Koc, G., \& Inan, L. E. (2017). The efficacy of greater occipital nerve blockade in chronic migraine: A placebo-controlled study. Acta Neurologica Scandinavica, 136(2), 138-144.

8. Headache Classification Committee of the International Headache Society (IHS). (2013). The international classification of headache disorders, (beta version). Cephalalgia, 33(9), 629808 .

9. Inan, L. E., Inan, N., Karadaş, Ö., Gül, H. L., Erdemoğlu, A. K., Türkel, Y., \& Akyol, A. (2015). Greater occipital nerve blockade for the treatment of chronic migraine: A randomized, multicenter, double-blind, and placebo-controlled study. Acta Neurologica Scandinavica, 132(4), 270-277.

10. Messlinger, K., Fischer, M. J., \& Lennerz, J. K. (2011). Neuropeptide effects in the trigeminal system: pathophysiology and clinical relevance in migraine. The Keio journal of medicine, 60(3), 82-89.

11. Noseda, R. and Burstein, R. (2013): Migraine pathophysiology: anatomy of the trigeminovascular pathway and associated neurological symptoms, cortical spreading depression, 
sensitization, and modulation of pain. Pain; 154: S44 -S53. 59(9): 1448-1467.

12. Olesen, J., Burstein, R., Ashina, M., \& Tfelt-Hansen, P. (2009). Origin of pain in migraine: evidence for peripheral sensitisation. The Lancet Neurology, 8(7), 679-690.

13. Soto, E., Bobr, V., \& Bax, J. A. (2012). Interventional techniques for headaches. Techniques in regional anesthesia and pain management, 16(1), 30-40.

14. Strassman, A. M., Raymond, S. A., \& Burstein, R. (1996). Sensitization of meningeal sensory neurons and the origin of headaches. Nature, 384(6609), 560-564.
15. VanderHoek, M. D., Hoang, H. T., \& Goff, B. (2013). Ultrasound-guided greater occipital nerve blocks and pulsed radiofrequency ablation for diagnosis and treatment of occipital neuralgia. Anesthesiology and pain medicine, 3(2), 256.

16. Zhang, H., Yang, X., Lin, Y., Chen, L., \& Ye, H. (2018). The efficacy of greater occipital nerve block for the treatment of migraine: a systematic review and meta-analysis. Clinical neurology and neurosurgery, 165, 129-133.
This work is licensed under Creative Commons Attribution 4.0 License

To Submit Your Article Click Here: Submit Manuscript

DOI: $10.31579 / 2578-8868 / 212$
Ready to submit your research? Choose Auctores and benefit from:

$>$ fast, convenient online submission

$>$ rigorous peer review by experienced research in your field

$>$ rapid publication on acceptance

$>$ authors retain copyrights

$>$ unique DOI for all articles

$>$ immediate, unrestricted online access

At Auctores, research is always in progress.

Learn more auctoresonline.org/journals/neuroscience-and-neurologicalsurgery 